Review

\title{
Review of Surface Plasmon Resonance and Localized Surface Plasmon Resonance Sensor
}

\author{
Yong CHEN and Hai MING \\ Department of Optics \& Optical Engineering, Anhui Key Laboratory of Optoelectronic Science and Technology, \\ University of Science and Technology of China, Hefei, Anhui, 230026, China \\ *Corresponding author: Hai MINGＥ-mail: minghai@ustc.edu.cn
}

\begin{abstract}
An overview of recent researches of surface plasmon resonance (SPR) sensing technology in Laboratory of Science and Technology of Micro-Nano Optics (LMNO), University of Science and Technology of China, is presented. Some novel SPR sensors, such as sensors based on metallic grating, metal-insulator-metal (MIM) nanoring and optical fiber, are designed or fabricated and tested. The sensor based on localized surface plasmon resonance (LSPR) of metallic nanoparticles is also be summarized. Because of the coupling of propagating surface plasmons and localized surface plasmons, the localized electromagnetic field is extremely enhanced, which is applied to surface-enhanced Raman scattering (SERS) and fluorenscence enhancement. Future prospects of SPR and/or LSPR sensing developments and applications are also discussed.
\end{abstract}

Keywords: Surface plasmons resonance, localized surface plasmon resonance, sensor, electromagnetic-field enhancement, high sensitivity

\section{Introduction}

Since the first application of surface plasmon resonance (SPR) phenomenon for gas detection and biologic sensor in 1982 [1], the SPR sensing technology has been widely used for the detection of biological and chemical analytes, environmental monitoring and medical diagnostics [2-5] in the past two decades.

Surface plasmons (SPs) are coherent oscillations of free electrons at the boundaries between metal and dielectric which are often categorized into two classes: propagating surface plasmons (PSPs) and localized surface plasmons (LSPs) [6]. PSPs can be excited on the metallic films which have several approaches as the Kretschman [7] and Otto [8] prism coupler, optical waveguides coupler [9], diffraction gratings [10], and optical fiber coupler [11], whereas LSPs can be excited on metallic nano-particles, which both can induce a strong enhancement of electromagnetic filed in the near-field region (resonance amplification), leading to a extensive application in surface-enhanced Raman scattering (SERS) [12], fluorescence enhancement [13], refractive index (RI) measurement [14], biomolecular interaction detection [15], and so on.

In this paper, we will review some recent works on SPR (based on PSPs) and localized surface plasmon resonance (LSPR) (based on LSPs) sensors at the Laboratory of Science and Technology of Micro-Nano Optics (LMNO) and make a prospect on the research and applications of SPR and LSPR sensors.

Received: 18 September 2011/ Revised version: 28 October 2011 


\section{Current research activities on SPR and LSPR sensors at LMNO}

Our research works are focused on new-style SPR sensors, bimetallic sensor chip for Kretschmann configuration, optical fiber SPR sensors and LSPR sensors on SERS and fluorescence enhancement.

\subsection{Novel-style SPR sensors}

Two types of SPR sensors with different styles are designed: metallic grating SPR sensor [16] and racetrack resonator SPR sensor [17]. Metallic grating SPR sensor has a high sensitivity for gas detection, and racetrack resonator SPR sensor has a broad linear detection range of analyte RI and high extinction ratio.

\subsubsection{Metallic grating SPR sensor}

We designed a highly sensitive grating-based SPR sensor for the gas detection [16]. The sensor has a high sensitivity at larger resonant incident angle if negative diffraction order of metallic grating is used to excite the surface plasmons, as shows in Fig. 1 .

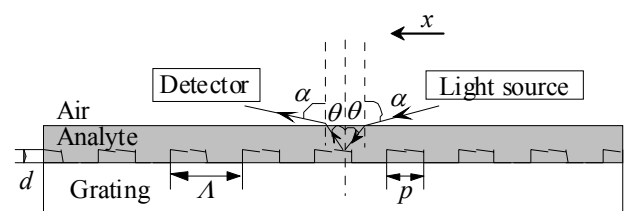

Fig. 1 Illustration of the SPR sensor based on metallic diffraction grating [16].

The resonant incident angle can be determined from the match condition of momentum at the interface of the metallic grating and analyte:

$$
\pm k_{0} \sqrt{\frac{\varepsilon_{m} n_{a}^{2}}{\varepsilon_{m}+n_{a}^{2}}}=k_{0} n_{a} \sin \theta_{\mathrm{res}}+m \frac{2 \pi}{\Lambda}
$$

where $\varepsilon_{m}$ is the permittivity of the metal, $\theta_{\text {res }}$ is the resonant angle of incidence, $n_{a}$ is the RI of analyte, $m$ is an integer representing the diffraction order, and sign "+" and sign "-" correspond to $m>0$ and $m<0$, respectively.

The sensitivity $\left(d \theta_{\text {res }} / d n\right)$ of the resonant angle of grating-based SPR sensors depends on the resonant incident angle (Fig. 2). The sensitivity of the negative diffraction $\operatorname{order}(m<0)$ is tens of times higher than that of positive diffraction order $(m>0)$ at large resonant angle, which is also much higher than that of conventional prism-based SPR sensor. For hydrogen detection, a thin $\mathrm{Pd}$ film is deposited on the metallic grating. When the Pd-coated gold grating is exposed to hydrogen with different concentrations, the permittivity of $\mathrm{Pd}$ layer will change. Then the change in the resonant angle can be detected. The theoretical resolution of hydrogen concentration of the order of $0.001 \%$ is obtained according to our design.

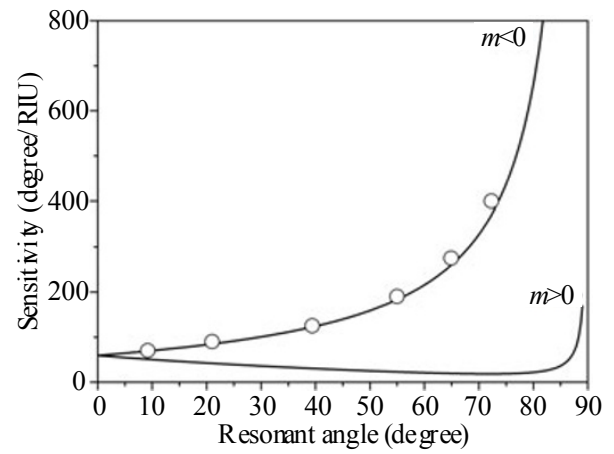

Fig. 2 Sensitivity of the resonant angle of grating-based SPR sensors versus the resonant angle of incidence $(\lambda=850 \mathrm{~nm}$, $\left.n_{a}=1.02\right)$ [16].

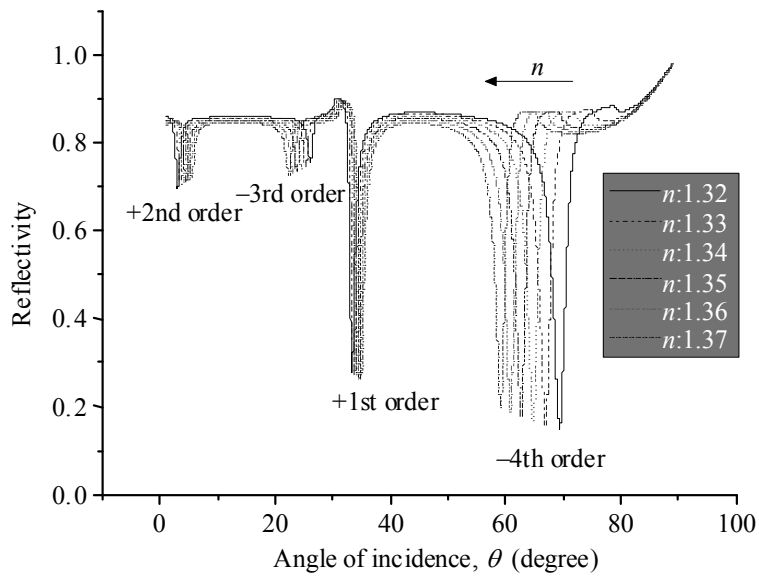

Fig. 3 Reflectance spectra of rectangle-grating-based SPR sensors with different analyte refractive indices [18].

The sensitivity of the metallic-grating-based SPR sensor can be improved by using double-dips method [18]. As shown in Fig.3, when the RI of the 
analyte changes, the resonant angle of negative diffraction order will change oppositely to that of positive diffraction order. The separation of negative diffraction order and positive diffraction order is detected to improve the sensitivity of metallic grating SPR sensors.

\subsubsection{SPR sensor based on racetrack resonator}

SPR sensing technique can be performed with an ultracompact racetrack resonator based on single mode metal-insulator-metal (MIM) plasmonic gap waveguide (Fig. 4).

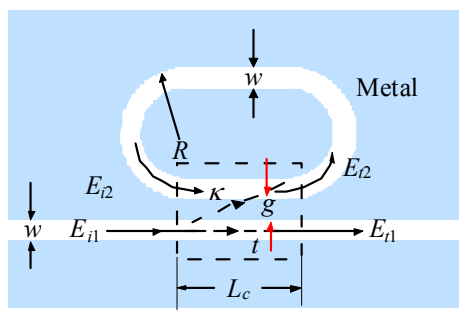

Fig. 4 Schematic of an MIM plasmonic racetrack resonator coupled to a straight waveguide [17].

The sensor is a compact and integratable one which consists an MIM racetrack resonator and a straight MIM waveguide as an evanescent wave coupler. A broad-banded light propagates along the straight MIM waveguide, and the light satisfying the match condition will be coupled into the surface plasmon mode of the MIM racetrack resonator. The transmitted spectrum detected at the other end of the straight MIM waveguide gives a harp dip at the resonant wavelength, and the resonant wavelength will red-shift as the RI of ambient material (the insulator in a racetrack resonator) increases (Fig. 5).

The theoretical sensitivity $\left(\Delta \lambda / \Delta n_{s}\right)$ of the racetrack resonator SPR sensor in this design is about $1030 \mathrm{~nm} /$ RIU. To achieve high coupling efficiency, the sensor should have a narrow resonant dip with a full width at half maximum (FWHM) of 9 $\mathrm{nm}$ and a high extinction ratio of $34.5 \mathrm{~dB}$. The "figure of merit" (FOM) [19] is defined as follows:

$$
F O M=\frac{\Delta \lambda / \Delta n_{s}}{F W H M} .
$$

The FOM of the racetrack resonator SPR sensors can be up to $114.4 \mathrm{RIU}^{-1}$.

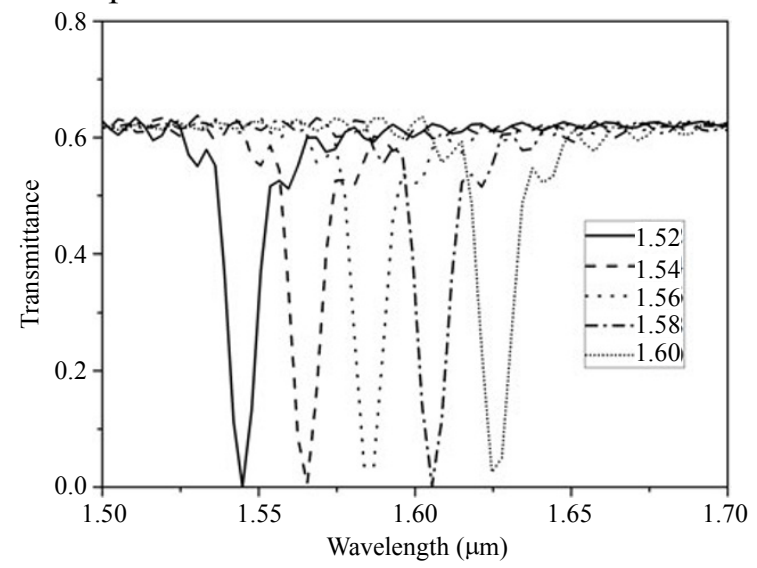

Fig. 5 Transmitted spectra with different analyte refractive indices [17].

\subsection{Traditional SPR sensors}

The traditional SPR sensor contains two different parts: Krestchmann prism coupler and optical fiber coupler. Firstly, we investigated the temperature effect on the SPR sensor of Krestchmann configuration [20]; then, a bimetallic SPR sensor chip is designed and tested in Biacore3000 for biomolecular detection [21]; after that, wavelength-modulated and intensity-modulated optical fiber SPR sensors are studied, respectively $[22,23]$.

\subsubsection{Temperature effect on the SPR sensor of Krestchmann configuration}

For actual applications of SPR sensor, ambient temperature will affect the measuring result [24]. As the most popular configuration, the effects of the temperature on an SPR sensor of Kretschmann configuration are investigated theoretically and experimentally.

Because of the change in properties of dielectric, metal, and analyte layers, the resonant dips are shifted and broadened at high temperature (Fig.6), which can be understood from the thermo-optic effect in the dielectric and analyte, together with a decrease of the plasmon frequency and an increase of the collision frequency in the metal layer when the temperature increases. 


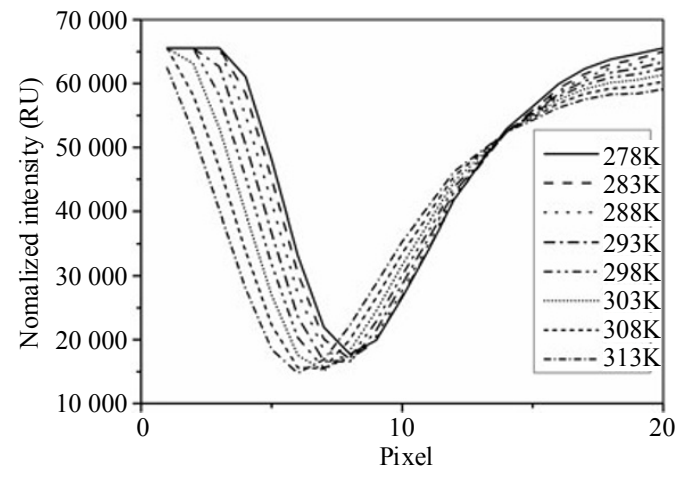

Fig. 6 Experimental SPR curves between normalized intensity and detective pixels at different temperatures [20].

A theoretical model is constructed by considering the temperature dependence of the properties of the metal, dielectric, and analyte layers. The numerical result is shown in Fig. 7, which is well consistent with the experimental result.

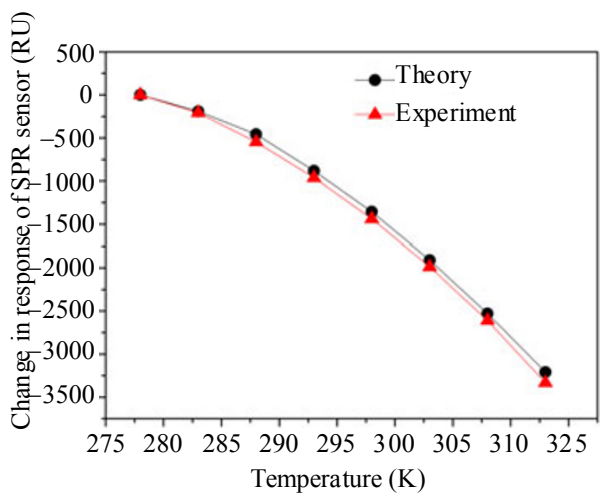

Fig. 7 Experimental and numerical results of the changes in response of the SPR sensor vary with temperatures [20].

The effects of the temperature from the sensor and the analyte are studied separately. The result shows that the thermal effect from the SPR sensor itself is much smaller than that of the aqueous analyte but comparable for the gas detection.

\subsubsection{Bimetallic SPR sensor chip}

Compared with traditional single gold films, the gold/silver bimetallic film has sharper SPR dip and higher sensitivity [25-27]. We deployed the bimetallic film into a commercial SPR instrument, Biacore3000 from GE Healthcare, to detect sulfamethoxazole (SMX), a kind of contaminations in food products, which is considered as a potential threat to consumers' health [28].

For the bimetallic sensor chip, a 40-nm silver film is deposited on the BK7 optical glass substrates, then, a 10-nm Au film is deposited on the 40-nm Ag film, which has the best FOM based on theoretical analysis.

Figure 8 presents the atomic force microscope (AFM) images and resonant dips of the gold film and the gold/silver bimetallic film, respectively. The surface roughness values of the single $\mathrm{Au}$ film and $\mathrm{Au} / \mathrm{Ag}$ bimetallic film (10-nm Au with 40-nm Ag) are $0.810 \mathrm{~nm}$ and $2.635 \mathrm{~nm}$, respectively. The roughness values of the $\mathrm{Au}$ film and bimetallic film are of the same order and are smooth enough for the SPR sensing experiments. The FWHM of the bimetallic layer is about $2 / 3$ times narrower than that of the single gold layer.

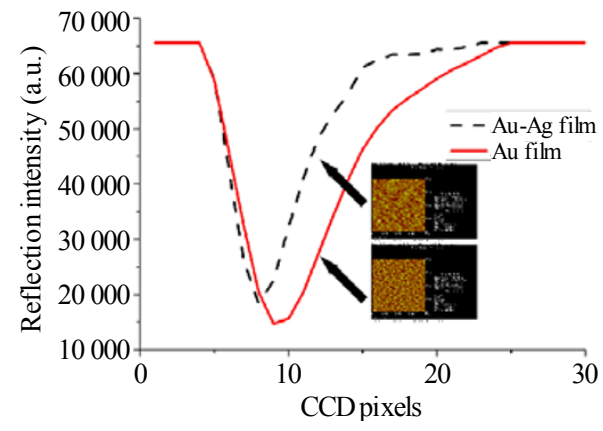

Fig. 8 AFM pictures and SPR curves of a single gold film sensor chip and gold/silver bimetallic sensor chip [21].

For actual testing, the analysis cycle contains of the binding of the anti-SMX with the SMX on the bimetallic chip and the regeneration of the bimetallic chip surface. The real-time monitoring of the sensing progress and the stability of the sensor are measured for 6 times, as shown in Fig. 9.

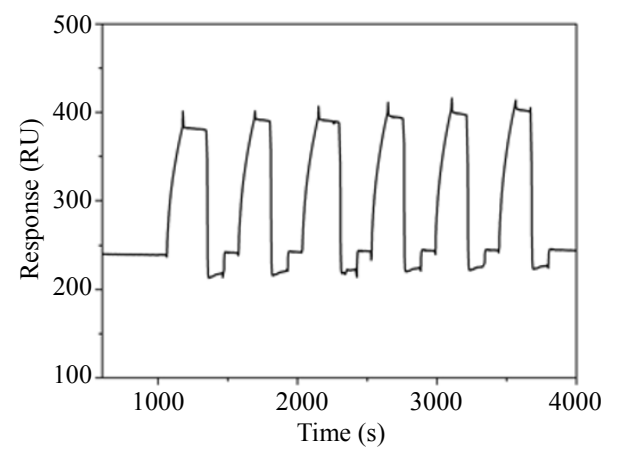

Fig. 9 Sensorgram from testing the bimetallic chip sensing progress for 6 times [21]. 
The limit of the detection of the SMX for the bimetallic film is $1.8 \mathrm{ng} / \mu \mathrm{l}$, which is lower than that of the single gold film $(3.2 \mathrm{ng} / \mu \mathrm{l})$ [29] by comparing to traditional single gold films.

\subsubsection{Optical fiber SPR sensors}

Optical fiber SPR sensors have been studied extensively based on their unique advantages such as miniaturization, high spatial resolution, and capability of on-line distributed measurement and remote sensing in dangerous environments [30, 31]. We designed and fabricated a novel wavelength-modulated optical fiber SPR sensor by using an ultra-thin dielectric film of $\mathrm{MgF}_{2}$ as the modulated and protected layer, the influences of the dielectric film on the sensitivity and shape of the resonant dip are investigated which are significant to the SPR fiber sensor.

To fabricate the optical fiber SPR sensor, 40-nm silver and $10-\mathrm{nm} \quad \mathrm{MgF}_{2}$ were homogeneously sputtered around the naked fiber core at a length of about $10 \mathrm{~mm}$ [core diameter: $200 \mu \mathrm{m}$, numerical aperture (NA): 0.37]. The structure of the sensor and the experimental sensing system are shown in Fig. 10.

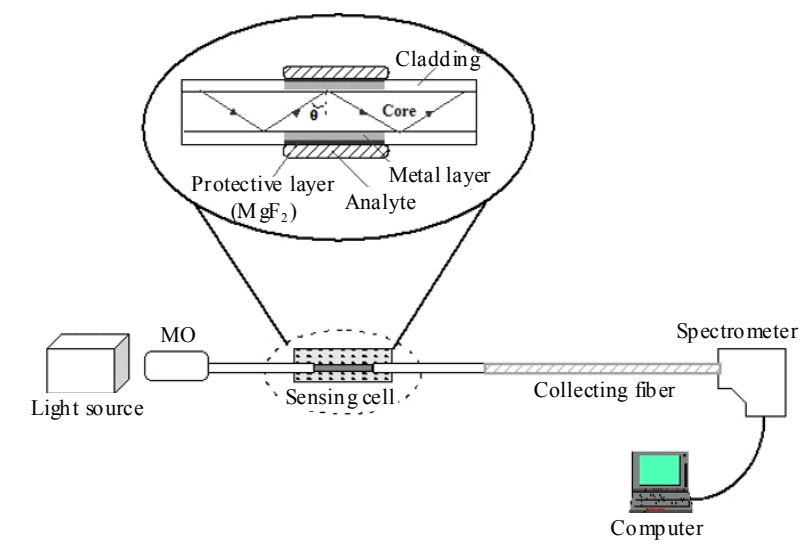

Fig. 10 Experimental setup of the optical fiber SPR sensor [22].

As shown in Fig. 11, the transmittance spectra shift to a longer wavelength when the analyte RI increases. When the analyte RI is tuned from 1.3419 to 1.3730 , the resonance wavelength varies from $552.7 \mathrm{~nm}$ to $631.2 \mathrm{~nm}$. The resonance peaks broaden at the high RI because of the higher absorption loss at the longer wavelength.

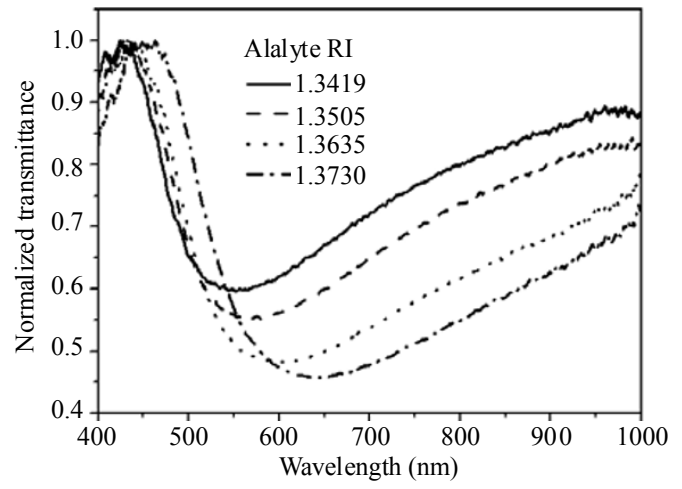

Fig. 11 Transmittance spectra for different analyte refractive indices of the sensor with the $\mathrm{MgF}_{2}$ film (Ag film: $40 \mathrm{~nm} ; \mathrm{MgF}_{2}$ film: $10 \mathrm{~nm})[22]$.

Figure 12 presents the simulated and experimental results of the SPR spectrum change with different analyte refractive indices for the sensor with and without the $\mathrm{MgF}_{2}$ film. When the liquid RI is lower (higher) than 1.38 (the RI of $\mathrm{MgF}_{2}$ ), the sensor with the $\mathrm{MgF}_{2}$ film has a longer(lower) resonance wavelength compared with the traditional structure (without $\mathrm{MgF}_{2}$ ) because the $\mathrm{MgF}_{2}$ film performs as a dielectric film that decreases (increases) the equivalent RI in the evanescent field. This is the modulation function of the dielectric film. Meanwhile, the sensitivity of the $\mathrm{MgF}_{2}$-protected fiber-optic SPR sensors increases monotonically with the analyte RI, which can reach $4464 \mathrm{~nm} / \mathrm{RIU}$ when the RI is 1.3945 .

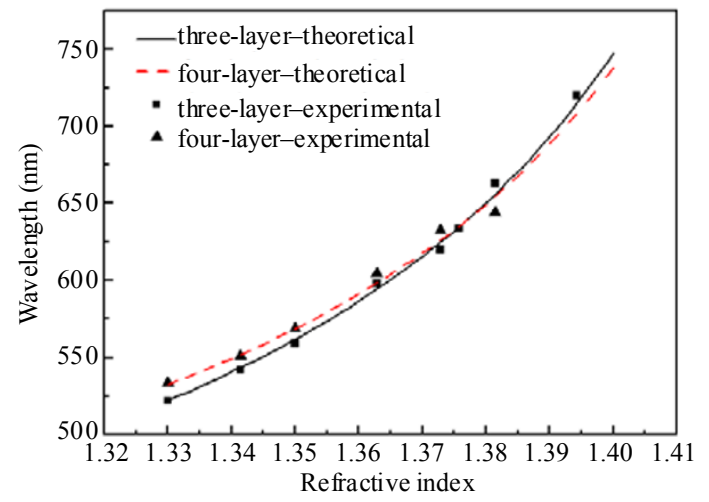

Fig. 12 Resonance wavelength vs the analyte RI (the dots are experimental results, and the lines are simulation results): the dashed line is the simulation curve for the sensor with the $\mathrm{MgF}_{2}$ film and the solid line is the simulation curve for the sensor without the $\mathrm{MgF}_{2}$ film [22]. 
For a similar structure but without the $\mathrm{MgF}_{2}$ film, the intensity-modulated optical fiber SPR sensor is studied with the radially polarized beam. Because of the rotational symmetry of the fiber, a radially polarized beam can improve the excite efficiency. The result is shown in Fig. 13. The sensitivity of the structure with the radially polarized beam is 3 times higher than that of using linearly polarized beam.

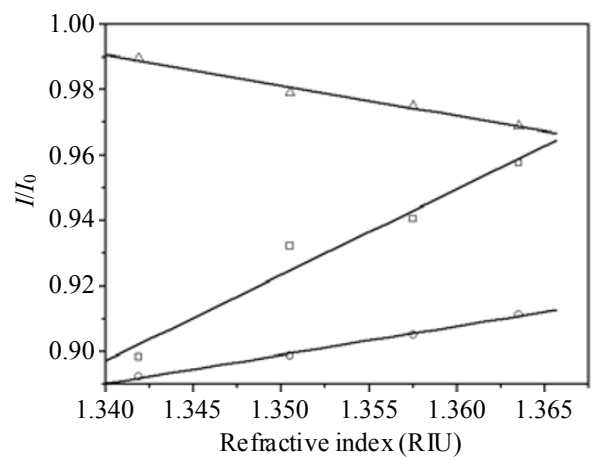

Fig. 13 Normalized transmitted light intensity vs refractive index (triangles: the results of the bare multi-mode fiber; circles: the results of the silver coated multi-mode fiber with the linearly polarized beam; squares: the results of the silver coated multi-mode fiber with the radially polarized beam [23].

\subsection{LSPR sensors}

Due to the localized surface plasmon resonance, the local electromagnetic field around the metallic nanoparitcles will be enhanced dramatically, and the resonant wavelength is sensitive to the variation of the local dielectric environment. Our researches in this area include LSPR for the RI measurement, LSPs in the optical fiber for SERS enhancement [32-34], coupling between LSPs/LSPs and LSPs/SPs for SERS enhancement and fluorescence enhancement [35-37].

\subsubsection{LSPR for RI measurement}

Noble metal nanoparticles, such as gold and silver, exhibit a strong optical extinction at visible and near-infrared (NIR) wavelengths and generate LSPR phenomenon which is sensitive to the surrounding medium. Gold nanorod is widely used for refractive index sensing because its longitudinal localized surface plasmon resonance is very sensitive to the ambient refractive index. For the experiment, gold nanorods are easily be fabricated by the seeded growth method [38], and its aspect ratio can also be tuned to have the proper resonant wavelength (Fig. 14).

Figure 14 illustrates the extinction spectra of two kinds of gold nanorods with different aspect ratios of 2.5:1 and 4.2:1. The longitudinal LSPR peak shifts to the longer wavelength as the refractive index increases, and the shift amplitude depends on the aspect ratio. The longer the aspect ratio is, the larger the shift will be. Thus we plot the longitudinal LSPR peak versus refractive index in Fig. 15 that shows nanorods with 4 different aspect ratios. It reveals an almost linear relationship between longitudinal LSPR peak and refractive index ranging from 1.33 to 1.47 in our experiment. As the aspect ratio varies from 2.5 to 4.2 , the sensitivity increases from $216 \mathrm{~nm} / \mathrm{RIU}$ to $352 \mathrm{~nm} / \mathrm{RIU}$.

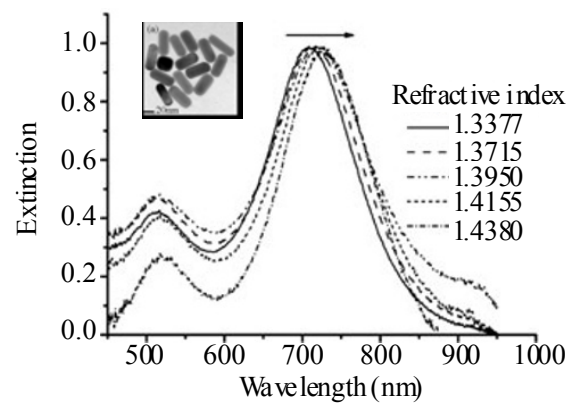

(a)

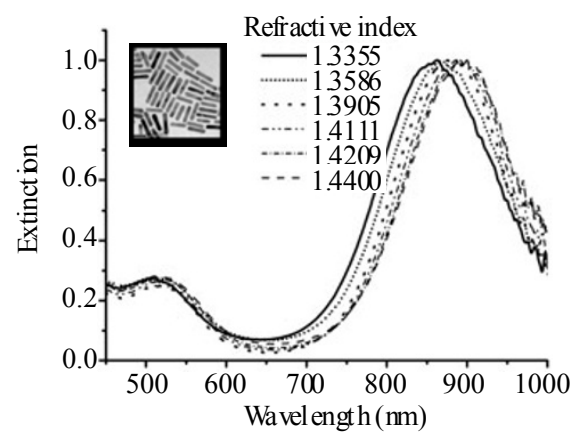

(b)

Fig. 14 TEM image of nanorods with the aspect ratio about 2.5:1 (a) and 4.2:1 (b), and the corresponding normalized extinction spectrum at various refractive indices. 


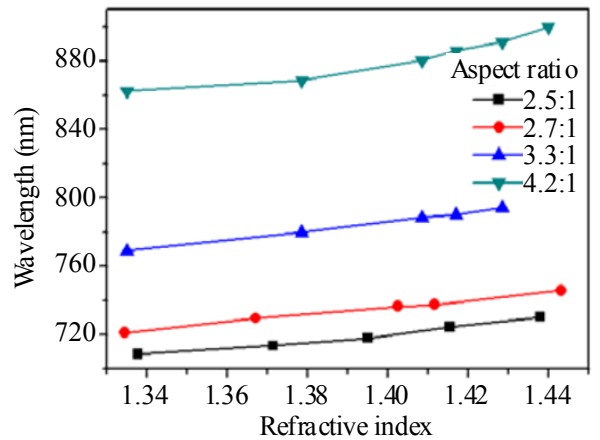

Fig. 15 Longitudinal LSPR peak versus refractive index for 4 different aspect ratios.

\subsubsection{Optical fiber SERS sensors}

Two different types of optical fiber are used in our experiment: polymer optical fiber (POF) and photonic crystal fiber (PCF). Compared with the silica fiber, POF shows a more flexible, effective and robust performance and better biocompatibility in the sensing [39-41] while PCF can provide very large internal surface area because of air-hole arrays for SERS action. A POF SERS sensor and a PCF SERS sensor are developed in our experiment as follows:

To fabricate the POF SERS sensor, we used the etching method [42] to prepare the POF tip with a diameter of $2 \mu \mathrm{m}-3 \mu \mathrm{m}$. Then several drops of gold nanorods colloids were placed directly on the POF tip and dried naturally. After that, the POF SERS probe was dipped into rhodamine 6G (R6G) solution $\left(10^{-9} \mathrm{M}\right)$ to detect the R6G SERS signal.

For the common "optrod" configuration [43], which the fiber is used to both transport the exciting laser radiation and collect the Raman scattering from the analyte, the Raman background of the POF would overwhelm the spectrum of the R6G dye. In this experiment, the light is focused on the tip directly with an inverted microscope objective, which is also used for collecting the signal. The spectra are achieved, as shown in Fig. 16. As a comparison, the same concentration analyte on the glass slide without gold nanorods is also measured. The SERS signal is enhanced 9-order magnitude which can be attributed to the local field of gold nanorods and their coupling.

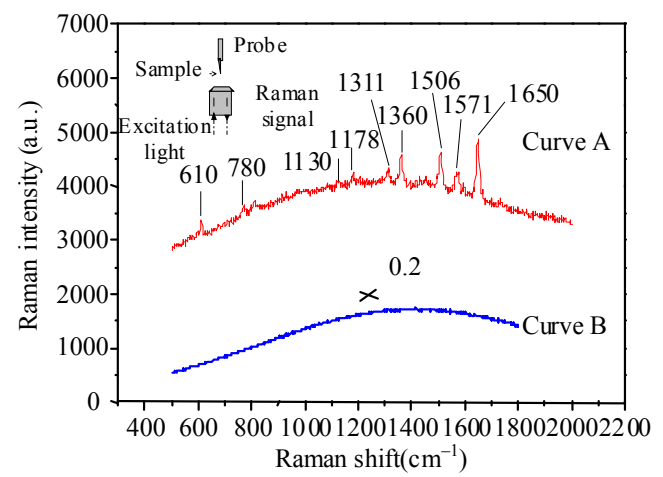

Fig. 16 Experimental schematic diagram for focusing light on the tip-Curve A: R6G molecules SERS signal obtained from the metal-coated taper tip; Curve B: signal from the same concentration R6G solution absorbed on glass slide without gold nanorods, both with excitation power $0.3 \mathrm{~mW}$, scan time $3 \mathrm{~s}$ [32].

Additionally, a broad spectral PCF SERS sensor is developed by using the solid core holey PCF with the silver nanoparticle cluster. A PCF SERS sensor is fabricated by injecting the mixture of the 4-mercaptobenzoic acid (4-MBA) aqueous solution with the silver colloid solution into the air holes of the PCF.

The experiment setup is shown in Fig. 17. A continuous-wave Ar-ion laser $(514.5 \mathrm{~nm})$ and a diode laser $(785 \mathrm{~nm})$ are used as the excitation source to verify the broad spectral characteristics of the SERS probe.

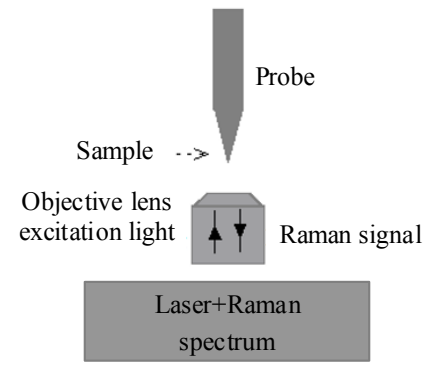

Fig. 17 Schematic diagram of the experimental setup [33].

As shown in Fig. 18, by using the visible light of $514.5 \mathrm{~nm}$ and near-infrared light of $785 \mathrm{~nm}$, the PCF SERS sensor has a good performance by obtaining an excellent and consistent SERS spectrum. Furthermore, the liquid holes' cladding results in a decrease of the numerical aperture and $\mathrm{V}$ parameter, 
which can be explained as that some high-order guide modes in air holes' cladding leak into the aqueous holes' cladding. Besides the low efficient way of the evanescent field, leakage modes can create important action in the solid core PCF SERS probe.

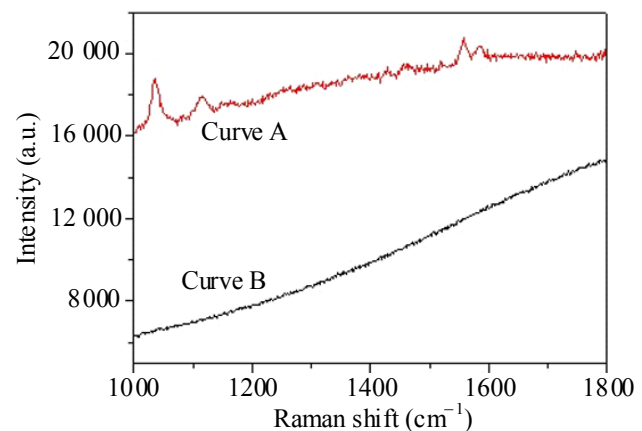

(a)

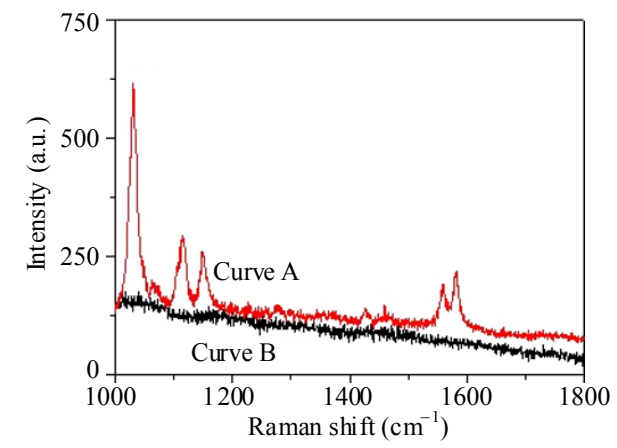

(b)

Fig. 18 Raman spectrum obtained from the 4-MBA with silver nanoparticles, excited by a $514.5-\mathrm{nm}$ (a) and a 785-nm laser (b): curve A with silver nanoparticles; curve B without silver nanoparticles [33].

\subsubsection{Coupling of LSPs in cube-array system for SERS enhancement}

To enhance the intensity and localization of the electromagnetic field, the coupling between LSPs is investigated [44, 45]. A quasi-three-dimensional system composed of silver nanocubes and silver nanohole arrays is fabricated (Fig. 19).

The silver nanohole arrays are thermally deposited on self-organized anodic aluminum oxide (AAO) substrates [46]. Then the R6G with a concentration of $5 \times 10^{-7} \mathrm{M}$ in the polymethylmethacrylate (PMMA) solution is spin-coated on the Ag hole arrays to form a spacer layer. Afterward $\mathrm{Ag}$ cubes are chemically synthesized by reducing $\mathrm{AgNO}_{3}$ with ethylene glycol [47-48] and dripped onto the R6G/PMMA layer. After drying at the room temperature for 24 hours, a coupled cube-hole array system is fabricated.

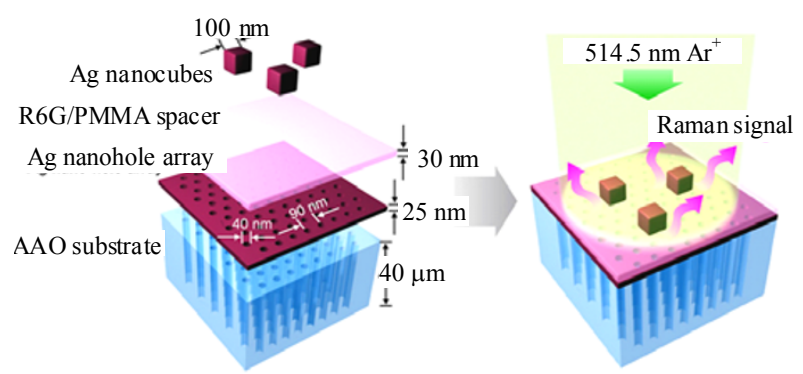

Fig. 19 Sketch of the coupled cube-hole array system [35].

Figure 20 presents the sketches of five types of structures and corresponding Raman spectra. Structure (d) is the coupled cube-hole array system with significant Raman signals. The enhancement factor (EF) [49] is of the order of $10^{8}$ which is more than one order of magnitude larger than that of other structures due to the large field enhancement of the "hot spots" induced by the plasmonic coupling between the silver cubes and silver hole arrays.

To further understand the coupling effect, series of tests are conducted as the following works to investigate the influence of each geometrical parameter in structure (d) (Fig. 21).

Cube-hole array distance: The EF decreases exponentially with an increase of distance due to the electrical field coupling decaying.

Hole diameter: the EF drops down steadily with an increase of the hole diameter because of the reducing effective coupling area between the $\mathrm{Ag}$ hole arrays and $\mathrm{Ag}$ cubes.

Inter-hole spacing: the EF decrease steadily with an increase of the inter-hole spacing because the closer the holes are, the more interaction between the LSPs is, which produce larger field enhancement.

Silver film thickness: the EF increases with a decrease of Ag film thickness. Because the thin film can enhance the interaction of the LSPs between the 
two sides which raise the local field intensity in the whole coupling region.

(a)

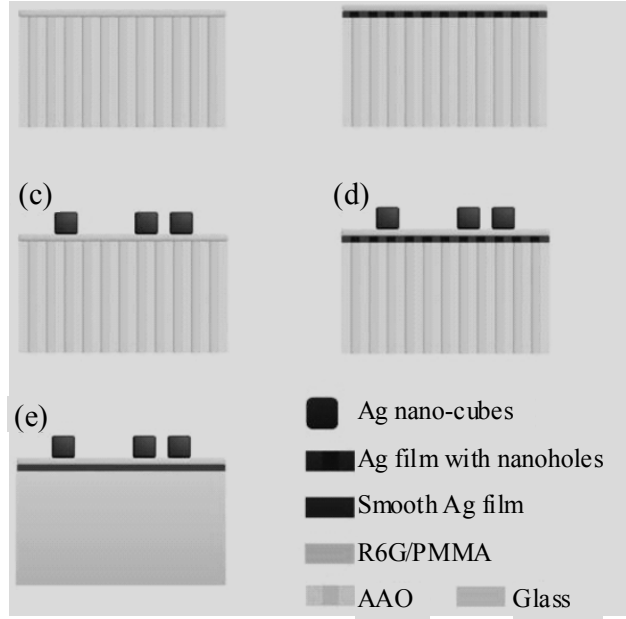

(f)

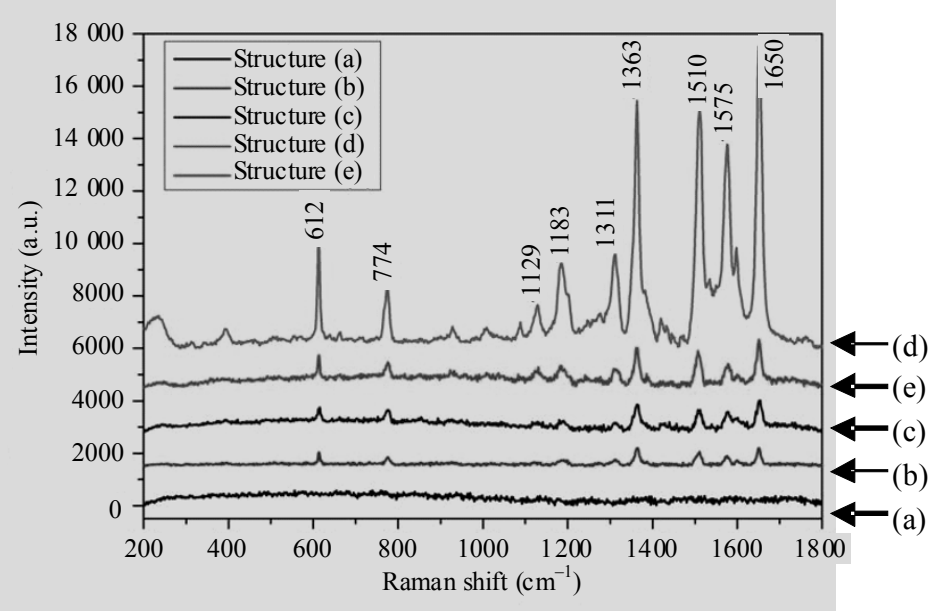

Fig. 20 Sketches of five types of samples: (a) none of the metallic components, (b) isolated silver hole array, (c) isolated silver cubes, (d) coupled silver cube-hole array structure [(a)-(d): all supported by the AAO substrate], (e) coupled silver nanocube-smooth film structure supported by glass, and (f) their corresponding Raman spectra [35].

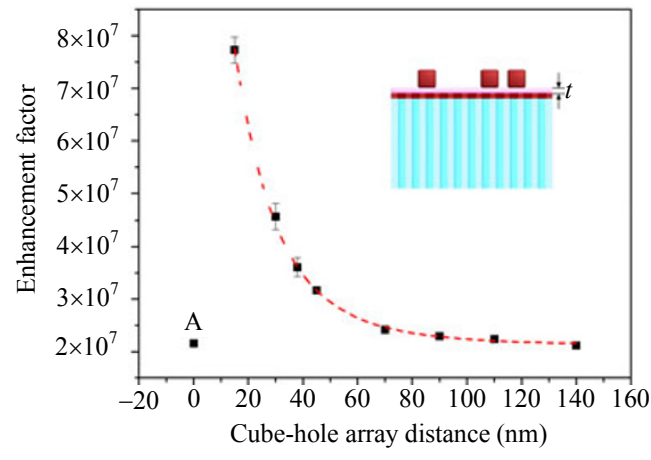

(a)

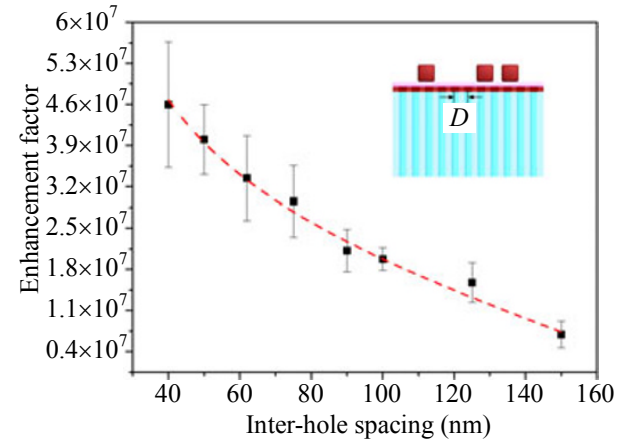

(c)

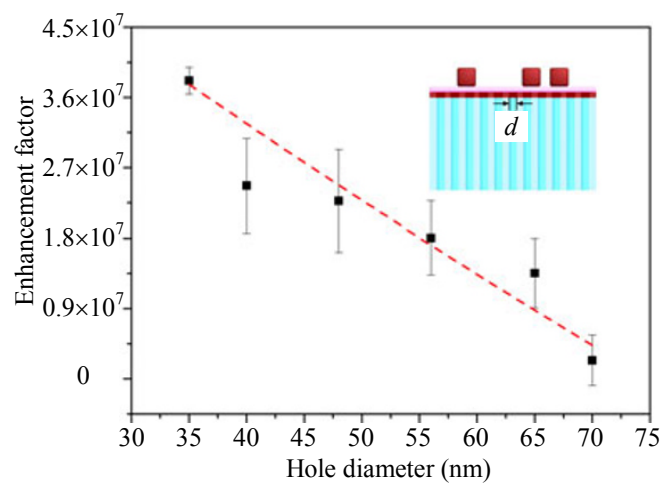

(b)

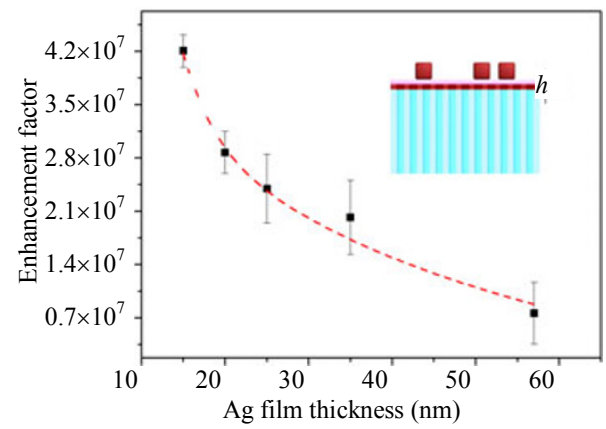

(d)

Fig. 21 SERS enhancement factors with different geometrical parameters [35].

\subsubsection{Coupling between LSPs and SPs for SERS enhancement and fluorescence enhancement}

The coupling between LSPs and SPs can strongly modify the electromagnetic field distribution, so the plasmonic interaction between silver nanocubes and a silver ground plane is studied for SERS and fluorescence enhancement.

In SERS experiment, a 57-nm-thick Ag film is 
evaporated on a glass substrate. PMMA films with different thicknesses are spin-coated onto Ag films. The Ag nanocubes are synthesized as mentioned above. With the mixture of a R6G solution and $\mathrm{Ag}$ colloid, the dyes will be adsorbed onto the cubes. Then the solution is dropped onto the PMMA film which forms the composite SERS substrate (S1) [Fig. 22(c)]. As a comparison, different substrates are fabricated: Ag nanocube/glass substrate (S2) and Ag nanocube/Ag film/glass substrate (S3) [Fig. 22 (a)].The Raman spectra are detected respectively in Figs. 22 (b) and 22(d).

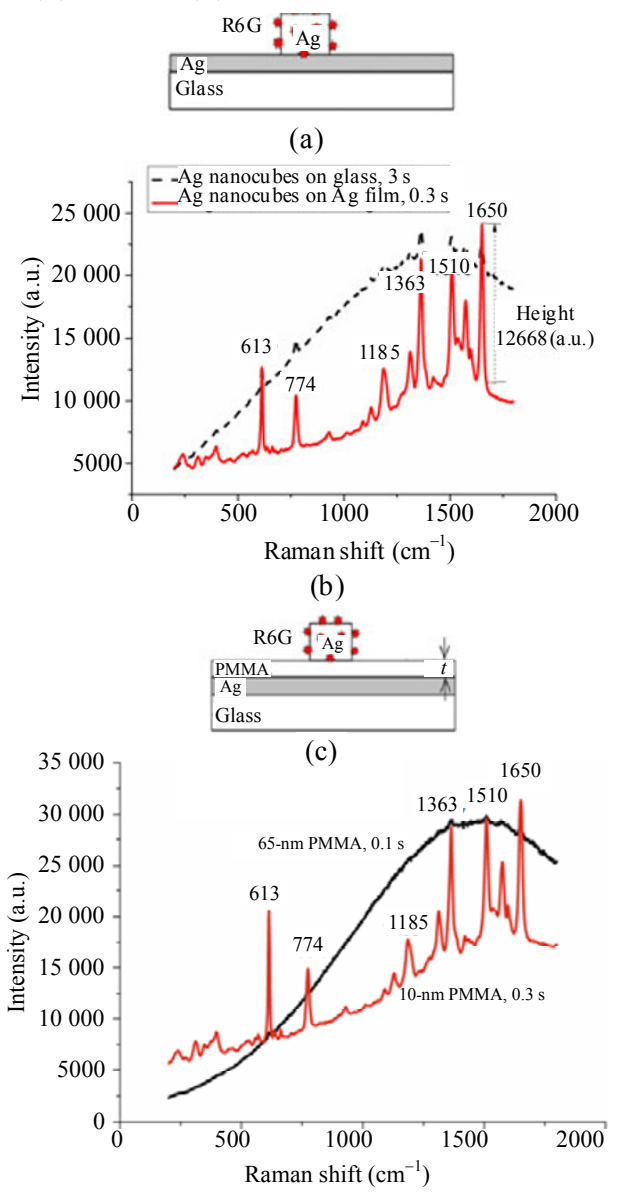

(d)

Fig. 22 (a) and (c) Sketch of the samples, (b) Raman spectra of R6G molecules on the silver film and glass substrates (the integrated time is $0.3 \mathrm{~s}$ and $3 \mathrm{~s}$, respectively), (d) Raman spectra of R6G molecules on the composite substrate with a spacer between the Ag nanocube and the Ag film (the thicknesses of the spacer layer are $10 \mathrm{~nm}$ and $65 \mathrm{~nm}$, respectively) [36].

By using this novel substrate, the Raman signal can be detected from R6G molecules with a solution concentration down to $10^{-11} \mathrm{M}$.

In the fluorescence enhancement experiment, the structure is similar to the structure mentioned above with only a little difference. The Rhodamine B (RhB) molecules are dropped in the PMMA as a spacer between the silver nanocubes and the silver film. The structure with no silver film is tested to make a comparison.

Figure 23 presents the fluorescence spectra from RhB-doped PMMA films for different structures. The experimental results show that the enhancement factor on the Ag film substrate can reach 521, which is much larger than that on the glass substrate.

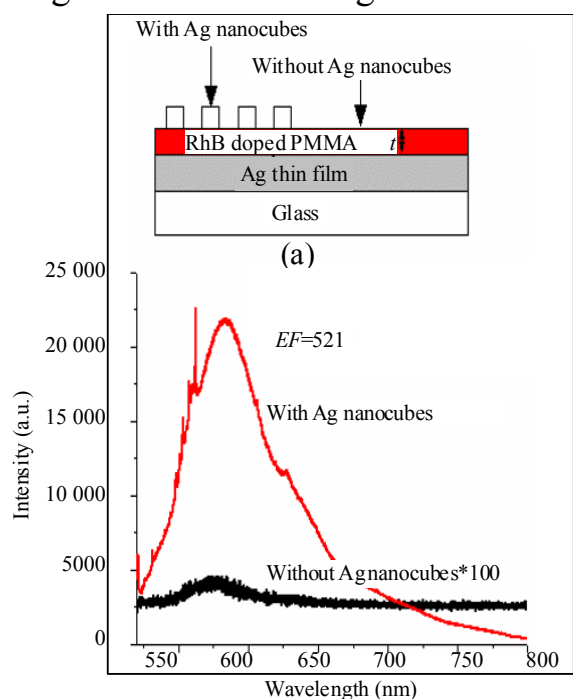

(b)

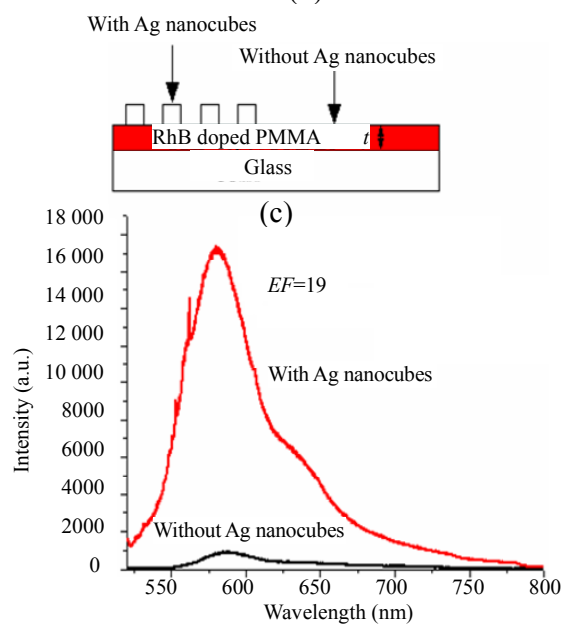

(d)

Fig. 23 (a) and (c) Sketch of the samples, (b) fluorescence spectra from RhB-doped PMMA films on silver film, and (d) fluorescence spectra from RhB-doped PMMA films on the glass substrate [37]. 


\section{Prospects of research on SPR and LSPR sensors}

As mentioned above, SPR and LSPR sensing technologies have a great application potentiality in many important areas including medicine, environmental monitoring, biotechnology, drug and food monitoring. But except for commercialized BIAcore and Texas Instrument sensors for biological sensing, other SPR and/LSPR sensing technologies still have not been deeply applied. To further overcome the limitation of the experimental condition and realize the extensive application, SPR/LSPR sensors have to be developed and improved to achieve more reliable requirements such as low cost, high sensitivity, facility, and robustness. Some future research works about SPR/LSPR can be expected in the following aspects:

(1) The investigation and realization of the novel-style SPR senor with the high sensitivity and high accuracy in the laboratory and practical application.

(2) The effect of the humidity, pressure, electric/magnetic field and other factors which would affect the performance of the SPR/LSPR sensor in actual applications.

(3) The investigation on the multi-channel optical fiber SPR sensor which can enhance the sensing efficiency and accuracy.

(4) The application and commercialization of the LSPR sensor based on SERS and fluorescence enhancement for the biological/chemical detection.

\section{Conclusions}

In summary, an overview on research of SPR and LSPR sensors in LMNO is presented in this paper. Novel style SPR sensors include metallic grating SPR sensor and racetrack resonator. The SPR sensor is designed and developed which demonstrate its unique advantages such as high sensitivity, miniaturization, and broad linear RI detection range. To improve the sensing performance of the traditional SPR sensor, temperature effect, we investigated both bimetallic sensor chip and dielectric film coated optical fiber SPR sensor numerically and experimentally. Furthermore, the LSPR sensing technology is studied and developed. The SERS and fluorescence enhancement of the LSPR shows its attractive potentiality for biological and chemical applications.

Over the past two decades, SPR and LSPR sensing technologies have being widely investigated, and a large number of SPR/LSPR sensor platforms, commercialized SPR biosensor, and measurement formats have been developed. The research works that are reviewed in this paper will directly contribute to the development and application of the SPR/LSPR sensing technologies.

\section{Acknowledgment}

This work is supported by the National Key Basic Research Program of China (No. 2011cb301802), and Key Program of National Natural Science Foundation of China (No. 60736037).

The authors gratefully acknowledge Prof. Y. H. Lu, D. G. Zhang, and P. Wang for many helpful discussions.

Open Access This article is distributed under the terms of the Creative Commons Attribution License which permits any use, distribution, and reproduction in any medium, provided the original author(s) and source are credited.

\section{References}

[1] B. Liedberg, C. Nylander, and I. Lunstrom, "Surface plasmon resonance for gas detection and biosensing," Sensors and Actuators, vol. 4, no. 2, pp. 299-304, 1983.

[2] R. C. Jorgenson and S. S. Yee, "A fiber-optic chemical sensor based on surface plasmon resonance," Sensors and Actuators B: Chemical, vol. 
12, no. 3, pp. 213-220, 1993.

[3] A. Huber, S. Demartis, and D. Neri, "The use of biosensor technology for the engineering of antibodies and enzymes," Journal of Molecular Recognition, vol. 12, no. 3, pp. 198-216, 1999.

[4] M. N. Weiss, R. Srivastava, H. Groger, P. Lo, and S. F. Luo, "A theoretical investigation of environmental monitoring using surface plasmon resonance waveguide sensors," Sensors and Actuators A: Physical, vol. 51, no. 2-3, pp. 211-217, 1995.

[5] D. R. Shankaran, K. V. Gobi, and N. Miura, "Recent advancements in surface plasmon resonance immunosensors for detection of small molecules of biomedical, food and environmental interest," Sensors and Actuators B: Chemical, vol. 121, no. 1, pp. 158-177, 2007.

[6] S. A. Maier, Plasmonics: Fundamentals and Applications. New York: Springer-Verlag, 2007, pp. 21-34.

[7] K. Matsubara, S. Kawata, and S. Minami, "Optical chemical sensor based on surface plasmon measurement," Applied Optics, vol. 27, no. 6, pp. 1160-1163, 1988.

[8] A. Otto, "Excitation of nonradiative surface plasma waves in silver by the method of frustrated total reflection," Zeitschrift für Physik A Hadrons and Nuclei, vol. 216, no. 4, pp. 398-410, 1968.

[9] R. D. Harris and J. S. Wilkinson, "Waveguide surface plasmon resonance sensors," Sensors and Actuators B: Chemical, vol. 29, no. 1-3, pp. 261-267, 1995.

[10] R. H. Ritchie, E. T. Arakawa, J. J. Cowan, and R. N. Hamm, "Surface-plasmon resonance effect in grating diffraction," Phys. Rev. Lett., vol. 21, no. 22, pp. 1530-1533, 1968.

[11] D. J. Webb, "Research activities arising from the University of Kent," Photonic Sensors, vol. 1, no. 2, pp. 140-151, 2011.

[12] C. Y. Chen and E. Burstein, "Giant Raman scattering by molecules at metal-island films," Phys. Rev. Lett., vol. 45, no. 15, pp. 1287-1291, 1980.

[13] K. Sokolov, G. Chumanov, and T. M. Cotton "Enhancement of molecular fluorescence near the surface of colloidal metal films," Anal. Chem., vol. 70, no. 18, pp. 3898-3905, 1998.

[14] J. Zeng, D. Liang, and Z. X. Cao, "Applications of optical fiber SPR sensor for measuring of temperature and concentration of liquids," in Proc. SPIE, vol. 5855, pp. 667-670, 2005.

[15] R. Karlsson and A. Fält, "Experimental design for kinetic analysis of protein-protein interactions with surface plasmon resonance biosensors," Journal of Immunological Methods, vol. 200, no. 1-3, pp. 121-133, 1998.

[16] K. Q. Lin, Y. H. Lu, J. X. Chen, R. S. Zheng, P. Wang, and H. Ming, "Surface plasmon resonance hydrogen sensor based on metallic grating with high sensitivity," Optics Express, vol. 16, no. 23, pp.
18599-18604, 2008.

[17] X. L. Wang, P. Wang, C. C. Chen, J. X. Chen, Y. H. Lu, H. Ming, and Q. W. Zhan, "Plasmonic racetrack resonator with high extinction ratio under critical coupling condition," Journal of Applied Physics, vol. 107, no. 12, pp. 124517-1-124517-4, 2010.

[18] D. B. Cai, Y. H. Lu, K. Q. Lin, P. Wang, and H. Ming, "Improving the sensitivity of SPR sensors based on gratings by double-dips method (DDM)," Optics Express, vol. 16, no. 19, pp. 14597-14602, 2008.

[19] L. J. Sherry, S. H. Chang, G. C. Schatz, R. P. Van Duyne, B. J. Wiley, and Y. Xia, "Localized surface plasmon resonance spectroscopy of single silver nanocubes," Nano. Lett., vol. 5, no. 10, pp. 2034-2038, 2005.

[20] K. Q. Lin, Y. H. Lu, Z. F. Luo, R. S. Zheng, P. Wang, and H. Ming, "Numerical and experimental investigation of temperature effects on the surface plasmon resonance sensor," Chinese Optics Letter, vol. 7, no. 5, pp. 428-431, 2009.

[21] Y. Chen, R. S. Zheng, D. G. Zhang, Y. H. Lu, P. Wang, H. Ming, Z. F. Luo, and Q. Kan, "Bimetallic chip for a surface plasmon resonance sensing instrument," Applied Optics, vol. 50, no. 3, pp. 387-391, 2011.

[22] Y. Chen, R. S. Zheng, Y. H. Lu, P. Wang, and H. Ming, "Fiber-optic surface plasmon resonant sensor with low-index anti-oxidation coating," Chinese Optics Letter, vol. 9, no. 10, pp. 100605-100608, 2011.

[23] J. Yan, Y. H. Lu, P. Wang, C. Gu, R. S. Zheng, Y. Chen, H. Ming, and Q. W. Zhan, "Improving the sensitivity of fiber-optic SPR sensor via radially polarized beam excitation," Chinese Optics Letter, vol. 7, no. 10, pp. 909-911, 2009.

[24] K. Q. Lin, L. M. Wei, D. G. Zhang, R. S. Zheng, P. Wang, Y. H. Lu, and H. Ming, "Temperature effects on prism-based surface plasmon resonance sensor," Chinese Physics Letters, vol. 24, no. 11, pp. 3081-3084, 2007.

[25] S. A. Zynio, A. V. Samoylov, E. R. Surovtseva, V. M. Mirsky, and Y. M. Shirshov, "Bimetallic layers increase sensitivity of affinity sensors based on surface plasmon resonance," Sensors, vol. 2, no. 2, pp. 62-70, 2002.

[26] X. C. Yuan, B. H. Ong, Y. G. Tan, R. Irawan, and S. C. Tjin, "Sensitivity stability optimized surface plasmon resonance sensing with double metal layers," J. Opt. A: Pure Appl. Opt., vol. 8, no. 11, pp. 959-963, 2006.

[27] Y. Y. Tan, X. C. Yuan, B. H. Ong, J. Bu, and Q. Y. Lin, "Two layered metallic film induced surface plasmons for enhanced optical propulsion of microparticles," Applied Physics Letter, vol. 91, no. 14, pp. 141108-1-141108-3, 2007. 
[28] S. Wang, H. Y. Zhang, L. Wang, Z. J. Duan, and I. Kennedy, "Analysis of sulphonamide residues in edible animal products: a review," Food Additives and Contaminants, vol. 23, no. 4, pp. 362-384, 2006.

[29] H. M. Zhou, H. C. OU, H. Jiang, H. F. Jiang, X. P. Wang, and Z. F. Luo, "Surface plasmon resonance for rapid determination of sulfamethoxazole in milk," Food Science, vol. 31, no. 6, pp. 168-171, 2010.

[30] A. K. Sharma, R. Jha, and B. D. Gupta, "Fiber-optic sensors based on surface plasmon resonance: a comprehensive review," IEEE Sensors Journal, vol. 7, no. 8, pp. 1118-1129, 2007.

[31] W. R. Habel and K. Krebber, "Fiber-optic sensor applications in civil and geotechnical engineering," Photonic Sensors, vol. 1, no. 3, pp. 268-280, 2011.

[32] Z. G. Xie, J. Tao, Y. H. Lu , K. Q. Lin, J. Yan, P. Wang, and H. Ming, "Polymer optical fiber SERS sensor with gold nanorods," Opt. Commun., vol. 282, no. 3, pp. 439-442, 2009.

[33] Z. G. Xie, Y. H. Lu, H. Wei, J. Yan, P. Wang, and H. Ming, "Broad spectral photonic crystal fiber surface enhanced Raman scattering probe," Applied Physics B, vol. 95, no. 4, pp. 751-755, 2009.

[34] Z. G. Xie, P. Wang, Y. H. Lu, K. Q. Lin, J. Yan, and H. Ming, "Photonic crystal fiber SERS sensors based on silver nanoparticle colloid," Chinese Physics Letters, vol. 25, no. 12, pp. 4473-4475, 2008.

[35] X. L. Wen, M. F. Yi, D. G. Zhang, P. Wang, Y. H. Lu, and H. Ming, "Tunable plasmonic coupling between silver nano-cubes and silver nano-hole arrays," Nanotechnology, vol. 22, no. 8, pp. 085203, 2011.

[36] M. F. Yi, D. G. Zhang, P. Wang, X. J. Jiao, S. Blair, X. L. Wen, Q. Fu, Y. H. Lu, and H. Ming, "Plasmonic interaction between silver nano-cubes and a silver ground plane studied by surface-enhanced Raman scattering," Plasmonics, vol. 6, no. 3, pp. 515-519, 2011.

[37] M. F. Yi, D. G. Zhang, X. L. Wen, Q. Fu, P. Wang, Y. H. Lu, and H. Ming, "Fluorescence enhancement caused by plasmonics coupling between silver nano-cubes and silver film," Plasmonics, vol. 6, no. 12, pp. 213-217, 2011.

[38] T. K. Sau and C. J. Murphy, "Seeded high yield synthesis of short au nanorods in aqueous solution," Langmuir, vol. 20, no. 15, pp. 6414-6420, 2004.

[39] F. Jimenez, J. Arrue, G. Aldabaldetreku, G. Durana,
J. Zubia, O. Ziemann, and C. A. Bunge, "Analysis ofa plastic optical fiber-based displacement sensor," Applied Optics, vol. 46, no. 25, pp. 6256-6262, 2007.

[40] C. M. Tay, K. M. Tan, S. C. Tjin, C. C. Chan, and H. Rahardjo, "Humidity sensing using plastic optical fibers," Microwave and Optical Technology Letters, vol. 43, no. 5, pp. 387-390, 2004.

[41] F. Baldini, P. Bechi, S. Bracci, F. Cosi, and F. Pucciani, "In vivo optical-fiber $\mathrm{pH}$ sensor for gastro-oesophageal measurements," Sensors and Actuators B: Chemical, vol 29, no. 1-3, pp. 164-168, 1995.

[42] D. F. Merchant, P. J. Scully, and N. F. Schmitt, "Chemical tapering of polymer optical fiber," Sensors and Actuators A: Physical, vol 76, no. 1-3, pp. 365-371, 1999.

[43] E. Polwart, R. L. Keir, C. M. Davidson, W. E. Smith, and D. A. Sadler, "Novel SERS-active optical fibers prepared by the immobilization of silver colloidal particles," Applied Spectroscopy, vol. 54, no. 4, pp. 522-527, 2000.

[44] P. K. Jain and M. A. El-Sayed, "Plasmonic coupling in noble metal nanostructures," Chem. Phys. Lett., vol. 487, no. 4-6, pp. 153-164, 2010.

[45] H. J. Chen, Z. H. Sun, W. H. Ni, K. C. Woo, H. Q. Lin, L. D. Sun, C. H. Yan, and J. F. Wang, "Plasmon coupling in clusters composed of two-dimensionally ordered gold nanocubes," Small, vol. 5, no. 18, pp. 2111-2119, 2009.

[46] H. Masuda and K. Fukuda, "Ordered metal nanohole arrays made by a two-step replication of honeycomb structures of anodic alumina," Science, vol. 268, no. 5216, pp. 1466-1468, 1995.

[47] S. E. Skrabalak, L. Au, X. Li, and Y. Xia, "Facile synthesis of Ag nanocubes and Au nanocages," Nat. Protocols, vol. 2, no. 9, pp. 2182-2190, 2007.

[48] A. R. Siekkinen, J. M. McLellan, J. Chen, and Y. Xia, "Rapid synthesis of small silver nanocubes by mediating polyol reduction with a trace amount of sodium sulfide or sodium hydrosulfide," Chem. Phys. Lett., vol. 432, no. 4-6, pp. 491-496, 2006.

[49] R. P. Van Duyne, J. C. Hulteen, and D. A Treichel, "Atomic force microscopy and surface-enhanced Raman spectroscopy. I. Ag island films and Ag film over polymer nanosphere surfaces supported on glass," J. Chem. Phys., vol. 99, no. 3, pp. 2101-2115, 1993. 